\title{
Artificial Immune Systems: structure, function, diversity and an application to biclustering
}

\author{
Leandro N. de Castro · Jon Timmis · Helder Knidel • \\ Fernando Von Zuben
}

Published online: 4 July 2009

(C) Springer Science+Business Media B.V. 2009

\section{Artificial Immune Systems}

The area of Artificial Immune Systems (AIS) is one of the most recent natural computing approaches and arose from the inter-disciplinary workings of immunologists, computer scientists, and engineers to develop solutions to such problems as distributed control and computer security. The field has developed significantly over the recent years, with the development of solutions to a wide variety of problems ranging from optimization, fault tolerance, data mining, bioinformatics, and robotic systems.

Within AIS, there are common, well understood algorithms, based on specific aspects of immunology. Clonal selection algorithms are the most widely employed, and take their inspiration from how cells of the adaptive immune system (specifically, cells called B-cells) interact with 'antigen' (something that causes an immune response) and then undergo a process of cloning, mutation, and selection. Negative selection algorithms, based on how immune cells (known as T-cells) are matured in a gland called the thymus are also

\footnotetext{
L. N. de Castro $(\bowtie)$

Mackenzie University, Rua da Consolação 896, Consolação, Sao Paulo, SP 01302-907, Brazil e-mail: lnunes@mackenzie.br

J. Timmis

Department of Computer Science, University of York, Heslington, York YO10 5DD, UK

e-mail: jtimmis@cs.york.ac.uk

J. Timmis

Department of Electronics, University of York, Heslington, York YO10 5DD, UK

H. Knidel

NatComp-From Nature to Business, R. do Comércio, 44, Center, Santos, SP 11010-140, Brazil e-mail: helder.knidel@natcomp.com.br

F. Von Zuben

Laboratory of Bioinformatics and Bio-inspired Computing (LBiC), School of Electrical and Computer Engineering (FEEC), University of Campinas (Unicamp), P.O. Box 6101, 13083-970 Campinas, SP, Brazil

e-mail: vonzuben@dca.fee.unicamp.br
} 
very popular. This process allows for the filtering of non-reactive cells in the immune system, and has been used, for example, as inspiration for building computer security systems. Finally, immune network algorithms, based on a theory regarding how immune cells (in this case B-cells) are maintained as memory in the immune system over time. These B-cells interact with each other in a stimulatory and inhibitory way to maintain the memory. This has been used as a metaphor to create dynamic learning algorithms and optimization methods.

\section{The contributions}

This special issue brings together extended papers from the 6th International Conference on Artificial Immune Systems, hosted in Santos, Brazil in August 2006. In ICARIS 2007, all participants received, together with the conference package, a Paper Evaluation Form to assess the Technical Quality and Presentation of each paper from the conference, divided into Student and Regular paper. From among the papers, three classes of awards were created: Best Conference Paper; Best Student Paper; and Best Presentation. A number of authors of these awarded papers were invited to substantially extend their papers for consideration in this special issue. After a detailed review process, three papers were selected to contribute to the special issue.

In the paper titled "Query Expansion Using an Immune-Inspired Biclustering Algorithm", authored by Pablo D. Castro; Fabricio de França; Hamilton Ferreira; Guilherme Coelho and Fernando Von Zuben, the authors proposed an immune-based biclustering algorithm to automatically add related terms to an information retrieval query. The idea is to cluster similar documents and assume that documents from the same cluster allow the finding of representative terms. The goal is to employ a biclustering algorithm to identify a set of relevant terms associated with each bicluster and use these terms to perform interactive query expansion. The algorithm, BIC-aiNet, was tested on two datasets, several queries were generated and the results were compared to those obtained by two proposals from the literature: the query expansion approach based on Term-Term Similarities (TTS) and the one based on the cosine similarity metric. The results showed that all algorithms returned unique relevant sets of suggestions to a given query, which appeared to be complementary in relation to the query expansion problem. The authors suggested that combining all approaches could lead to an increase in Recall, without substantially decreasing Precision.

Emma Hart, Hugues Bersini and Francisco Santos presented a work titled "Structure vs Function: A Topological Perspective on Immune Networks". The authors argue that the global properties of a network rely largely upon the relationship between its structure and function, what has been attracting much attention nowadays due to the Complex Network theory. The ubiquity of some classes of networks raises questions about the origin of specific topological properties and is useful for the understanding of complex social and biological phenomena. They introduced the notion of the 'potential network', which enables a direct study of the influence of different topologies on network behavior. This mechanism allows topological characteristics to be isolated to study the relationship between topology, dynamics, and functionality, thus allowing generic hypotheses to be proposed and tested, which are not restricted to a particular shape-space or affinity function or to any particular type of model dynamics.

Finally, in the work titled "Neural Network Ensembles: Immune-Inspired Approaches to the Diversity of Components", authored by Rodrigo Pasti, Leandro N. de Castro, 
Guilherme P. Coelho, and Fernando Von Zuben, the authors applied two immune-inspired algorithms, namely opt-aiNet and omni-aiNet, to train multi-layer perceptrons (MLPs) to be used in the construction of ensembles of classifiers. The authors investigated the diversity of the solutions generated by each of these algorithms and how these influence the performance of ensembles of classifiers. The results presented showed that the diversity of the components influences the quality of the ensembles, but it should be allied with high quality classifiers in order to lead to a significant improvement in the ensembles performance. 